\title{
Liouvillian integrability of a general Rayleigh-Duffing oscillator
} Jaume Giné, Claudia Valls

To cite this article: Jaume Giné, Claudia Valls (2019) Liouvillian integrability of a general Rayleigh-Duffing oscillator, Journal of Nonlinear Mathematical Physics 26:2, 169-187, DOI: https://doi.org/10.1080/14029251.2019.1591710

To link to this article: https://doi.org/10.1080/14029251.2019.1591710

Published online: 04 January 2021 


\title{
Liouvillian integrability of a general Rayleigh-Duffing oscillator
}

\author{
Jaume Giné \\ Departament de Matemàtica, Inspires Research Centre, Universitat de Lleida \\ Avda. Jaume II, 69; 25001 Lleida, Catalonia, Spain \\ gine@matematica.udl.cat \\ Claudia Valls \\ Departamento de Matemática, Instituto Superior Técnico \\ Av. Rovisco Pais 1049-001, Lisboa, Portugal \\ cvalls@math.ist.utl.pt
}

Received 4 May 2018

Accepted 15 October 201

\begin{abstract}
We give a complete description of the Darboux and Liouville integrability of a general Rayleigh-Duffing oscillator through the characterization of its polynomial first integrals, Darboux polynomials and exponential factors.

Keywords: Liouvillian integrability; Rayleigh-Duffing oscillator; first integrals.
\end{abstract}

2010 Mathematics Subject Classification: 34A05, 34C05, 37C10

\section{Introduction and statement of the main results}

We will consider the polynomial differential system

$$
\begin{aligned}
& \dot{x}=y, \\
& \dot{y}=a x+b y+c x^{3}+d y^{3},
\end{aligned}
$$

where $a, b, c, d \in \mathbb{R}$ are arbitrary parameters and $c^{2}+d^{2} \neq 0$ (otherwise the system is linear). When $c=d=-1$ this system is the well-known Rayleigh-Duffing oscillator introduced by Rayleigh in [15] (in that case, $x$ represents the displacement, $a$ is related with the stiffness and $b$ with the damping) and commonly studied from the dynamical point of view in the references $[3,7,8]$. Note that system (1.1) is invariant by the symmetry $(x, y) \mapsto(-x,-y)$.

For a deterministic system, the existence of constants or integrals of motion are responsible for the regular evolution of the phase-space trajectories of the system in well-defined regions of the phase space. For a planar differential system the existence of integrals of motion (called below first integrals) imply the regular evolution of the phase-space trajectories of the system in well-defined regions of the phase space and so the system can be integrated, at least qualitatively, since it can be determined theoretically its phase portrait, see [1]. However, for a differential system it is a difficult problem to determine the existence of integrals of motion (or first integrals). We recall that a function $H(x, y)$ is a first integral of a planar differential if it is a $C^{1}$ function defined on a full Lebesgue measure subset $U \subseteq \mathbb{R}^{2}$ which is constant along each orbit in $U$ of that system but it is not locally constant on any positive Lebesgue measure subset of $U$. 
When the differential system is polynomial, we can use the Darboux theory of integrability because it provides conditions (that are sufficient) to obtain integrability inside the family of Liouvillian functions (i.e., functions obtained from complex rational functions by a finite process of integrations, exponentiations and algebraic operations). This will be our main tool in the paper. The Darboux theory of integrability can be applied to real or complex polynomial differential systems, however the study of complex algebraic solutions is necessary for obtaining the real first integrals of any real polynomial differential system. In section 2 we have included all the results related with the Darboux theory of integrability that are needed in the paper.

The main result in the work is the following:

Theorem 1.1. The following holds for system (1.1) with $c^{2}+d^{2} \neq 0$ :

(a) If $d=0$ and $c \neq 0$ it is Liouville integrable if and only if either $b=0$ or $a=-2 b^{2} / 9$.

(b) If $d \neq 0$ and $c=0$ it is Liouville integrable if and only if $a=0$.

(c) If $c d \neq 0$ it is not Liouville integrable.

The proof of statements (a) and (b) of Theorem 1.1 is given in section 3 and the proof of Theorem 1.1 (c) is given in section 4 . In section 2 we have included all the auxiliary results that will be needed to prove Theorem 1.1. The proof of statement (c) of Theorem 1.1 corrects statement (b) and some gaps in the proof of Theorem 1 in [6].

\section{Preliminary results}

Consider a polynomial differential system of degree $d \in \mathbb{N}$

$$
\dot{\mathbf{x}}=P(\mathbf{x}), \quad \mathbf{x}=\left(x_{1}, x_{2}\right) \in \mathbb{R}^{2},
$$

where $P(\mathbf{x})=\left(P_{1}(\mathbf{x}), P_{2}(\mathbf{x})\right), P_{i} \in \mathbb{C}[\mathbf{x}], d=\max \left\{\operatorname{deg} P_{1}, \operatorname{deg} P_{2}\right\}$ is the degree of system (2.1) and the dot denotes derivative with respect to the independent variable $t$.

Let $\mathscr{X}$ be the vector field associated with system (2.1), i.e.,

$$
\mathscr{X}=P(\text { mathbfx })=\left(P_{1}\left(x_{1}, x_{2}\right), P_{2}\left(x_{1}, x_{2}\right)\right) .
$$

If $H$ is a first integral, then

$$
\mathscr{X}(H)=P_{1} \frac{\partial H}{\partial x_{1}}+P_{2} \frac{\partial H}{\partial x_{2}}=0 .
$$

A Darboux polynomial of system (2.1) is a polynomial $f \in \mathbb{C}[\mathbf{x}]$ such that

$$
\mathscr{X}(f)=P_{1} \frac{\partial f}{\partial x_{1}}+P_{1} \frac{\partial f}{\partial x_{2}}=K f
$$

where $\mathbf{x}=\left(x_{1}, x_{2}\right), K \in \mathbb{C}[\mathbf{x}]$ and has degree at most $d-1$. The polynomial $K$ is said to be the cofactor. An invariant algebraic curve is a curve given by $f=0$ such that $f \in \mathbb{C}[\mathbf{x}]$.

An exponential factor of system (2.1) is a function $E=\exp (g / f)$, with $f, g \in \mathbb{C}[\mathbf{x}]$ being coprime, such that

$$
\mathscr{X}(E)=P_{1} \frac{\partial E}{\partial x_{1}}+P_{2} \frac{\partial E}{\partial x_{2}}=L E
$$

where $L \in \mathbb{C}[\mathbf{x}]$ and has degree at most $d-1$. It is known that either $f$ is constant, or $f$ is a Darboux polynomial of system (2.1) and $\mathscr{X}(g)=K g+L f$, where $K$ is the cofactor of $f$. 
An inverse integrating factor of system (2.1) is a function $V$ such that

$$
\mathscr{X}(V)=P_{1} \frac{\partial V}{\partial x_{1}}+P_{2} \frac{\partial V}{\partial x_{2}}=\left(\frac{\partial P_{1}}{\partial x_{1}}+\frac{\partial P_{2}}{\partial x_{2}}\right) V .
$$

When $V \in \mathbb{C}\left[x_{1}, x_{2}\right]$, it is a Darboux polynomial whose cofactor is the divergence of the system.

We use the following result in [5] for finding Liouville first integrals.

Theorem 2.1. If system (2.1) of degree $d$ has $p$ Darboux polynomials $f_{i}$ with cofactors $K_{i}, i=$ $1, \ldots, p$, and $q$ exponential factors $E_{j}=\exp \left(g_{j} / h_{j}\right)$ with $L_{j}, j=1, \ldots, q$, then there exist $\alpha_{i}, \beta_{j} \in \mathbb{C}$ not all zero such that

$$
\sum_{i=1}^{p} \alpha_{i} K_{i}+\sum_{j=1}^{q} \beta_{j} L_{j}=\operatorname{div}(\mathscr{X})
$$

if and only if the function

$$
f_{1}^{\alpha_{1}} \cdots f_{p}^{\alpha_{p}} E_{1}^{\beta_{1}} \cdots E_{q}^{\beta_{q}}
$$

(called Darboux function) is an inverse integrating factor of $\mathscr{X}$. Here $\operatorname{div}(\mathscr{X})$ stands for the divergence of the system.

To prove the results related with the Liouville integrability we will use the following result that it is proved in [14].

Theorem 2.2. System (2.1) has a Liouville first integral if and only if it has an integrating factor which is a Darboux function (see (2.4)).

Note that the previous theorem states that with the Darboux method one can find all Liouville first integrals.

Let $J$ be the Jacobian matrix of $\mathscr{X}$. The following proposition was proved in $[12,13]$ we state it as we will use it. We recall that a polynomial first integral is a first integral that is a polynomial.

Theorem 2.3. Assume that the eigenvalues of the Jacobian matrix of $\mathscr{X}$ at some singularity $\left(\bar{x}_{1}, \bar{x}_{2}\right)$ satisfy $k_{1} \lambda_{1}+k_{2} \lambda_{2} \neq 0$ for $k_{1}, k_{2} \in \mathbb{Z}^{+}$and $k_{1}+k_{2} \geq 1$. Then $\mathscr{X}$ has no polynomial first integral.

The following theorem is due to Li et al. in $[9,10]$.

Theorem 2.4. Assume that the eigenvalues of the Jacobian matrix of $\mathscr{X}$ at some singularity $\left(\bar{x}_{1}, \bar{x}_{2}\right)$ satisfy $\lambda_{1}=0$ and $\lambda_{2} \neq 0$. Then $\mathscr{X}$ can have a polynomial first integral if and only if $\left(\bar{x}_{1}, \bar{x}_{2}\right)$ is nonisolated.

The singularities appearing in Theorems 2.3 and 2.4 can be real or complex, but system (2.1) is always real.

Let $\tau: \mathbb{C}^{2}\left[x_{1}, x_{2}\right] \rightarrow \mathbb{C}^{2}\left[x_{1}, x_{2}\right]$ be the automorphism defined by

$$
\tau\left(x_{1}, x_{2}\right)=\left(-x_{1},-x_{2}\right)
$$

and for a polynomial $p\left(x_{1}, x_{2}\right)$ let $\tau_{*}(p)=p\left(\tau\left(x_{1}, x_{2}\right)\right)$.

Proposition 2.1. If $g$ is an irreducible Darboux polynomial of degree d of system (2.1) with cofactor $K\left(x_{1}, x_{2}\right)$, then $f=g \cdot \tau_{*}(g)$ is also a Darboux polynomial invariant by $\tau$ with a cofactor of the form $K+\tau_{*}(K)=K\left(x_{1}, x_{2}\right)+K\left(-x_{1},-x_{2}\right)$. 
Proof. The proposition follows easily if we show that $\tau_{*}(g)$ is a Darboux polynomial with cofactor $\tau_{*}(K)$. To do it, note that applying $\tau_{*}$ to $(2.2)$ we get

$$
\tau_{*}(\mathscr{X}(g))=\tau_{*}(K g)
$$

Moreover, using that $\tau^{-1}=\tau$ we obtain

$$
\tau_{*}(\mathscr{X}(g))=\mathscr{X}\left(g \circ \tau^{-1}\right)=\mathscr{X}(g \circ \tau)=\mathscr{X}\left(\tau_{*}(g)\right) .
$$

On the other hand,

$$
\tau_{*}(K g)=(K g) \circ \tau^{-1}=(K g) \circ \tau=(K \circ \tau)(g \circ \tau)=\tau_{*}(K) \tau_{*}(g)
$$

Using (2.6), (2.7) and (2.8) we conclude that

$$
\mathscr{X}\left(\tau_{*}(g)\right)=\tau_{*}(K) \tau_{*}(g)
$$

as we wanted to prove.

The following result was proved in [2] (see Theorems 13 and 14). We recall that two real numbers $\omega_{1}, \omega_{2}$ are said to be rationally independent if none of them can be written as a linear combination of the other with rational coefficients, or in other words, if the only integers $k_{1}, k_{2}$ such that $k_{1} \omega_{1}+k_{2} \omega_{2}=0$ is $k_{1}=k_{2}=0$.

Proposition 2.2. Let $\left(\bar{x}_{1}, \bar{x}_{2}\right)$ be a singularity of system (2.1) and $\lambda_{1}, \lambda_{2}$ be the eigenvalues of the Jacobian matrix at $\left(\bar{x}_{1}, \bar{x}_{2}\right)$. Assume that $f\left(x_{1}, x_{2}\right)=0$ is an irreducible invariant algebraic curve with cofactor $K\left(x_{1}, x_{2}\right)$. If $f\left(\bar{x}_{1}, \bar{x}_{2}\right) \neq 0$ then $K\left(\bar{x}_{1}, \bar{x}_{2}\right)=0$. Moreover if $f\left(\bar{x}_{1}, \bar{x}_{2}\right)=0, \lambda_{2} \neq 0$ and either $\lambda_{1}$ and $\lambda_{2}$ are rationally independent, or $\lambda_{1} \lambda_{2}<0$, then $K\left(\bar{x}_{1}, \bar{x}_{2}\right) \in\left\{\lambda_{1}, \lambda_{2}, \lambda_{1}+\lambda_{2}\right\}$.

A polynomial $f\left(x_{1}, x_{2}\right)$ is said to be a weight homogeneous polynomial if there exist $s=$ $\left(s_{1}, s_{2}\right) \in \mathbb{N}^{2}$ and $m \in \mathbb{N}$ such that for all $\alpha \in \mathbb{R} \backslash\{0\}$,

$$
f\left(\alpha^{s_{1}} x_{1}, \alpha^{s_{2}} x_{2}\right)=\alpha^{m} f\left(x_{1}, x_{2}\right),
$$

where $\mathbb{R}$ denotes the set of real numbers, and $\mathbb{N}$ the set of positive integers. We shall refer to $s=$ $\left(s_{1}, s_{2}\right)$ as the weight of $f, m$ as the weight degree, and $x=\left(x_{1}, x_{2}\right) \mapsto\left(\alpha^{s_{1}} x_{1}, \alpha^{s_{2}} x_{2}\right)$ as the weight change of variables.

\section{Proof of statements (a) and (b) of Theorem 1.1}

Statement (a) of Theorem 1.1 is a consequence of Theorem 1.7 in [4]. In [4] are used the Puiseux series (see [4] for its definition) to find the Liouville integrability. In fact we have checked her results using the methods described in the present work and we have found the same results for the case $d=0$ and $c \neq 0$. Moreover the case $d=b=0$ with $c \neq 0$ corresponds to the Hamiltonian system $\dot{x}=y, \dot{y}=a x+c x^{3}$ which has the polynomial first integral $H=-2 a x^{2}-c x^{4}+2 y^{2}$ and the case 
$d=0, a=-2 b^{2} / 9$ with $c \neq 0$ is Liouville integrable because it has the inverse integrating factor

$$
V=\left(2 b^{2} x^{2}-9 c x^{4}-12 b x y+18 y^{2}\right)^{3 / 4} .
$$

Now we prove Theorem 1.1 (b). First note that if $a=0$ then system (1.1) becomes

$$
\dot{x}=y, \quad \dot{y}=-b y-d y^{3} .
$$

This system has the inverse integrating factor $V=b y+d y^{3}$ and in view of Theorems 2.1 and 2.2 it is Liouvillian integrable.

Now we consider $a \neq 0$. Statement (b) of Theorem 1.1 will now be an immediate consequence of the following proposition.

Proposition 3.1. System (1.1) with $c=0$ and $a \neq 0$ is not Liouvillian integrable.

Proof. Let $c=0$ and $a \neq 0$. We recall that since $c=0$ then $d \neq 0$. With the change of variables

$$
x \rightarrow \frac{1}{d B} x_{1}, \quad y \rightarrow B y_{1}, \quad s \rightarrow B^{2} d t, \quad B= \begin{cases}\left(a / d^{2}\right)^{1 / 4}, & a>0 \\ \left(-a / d^{2}\right)^{1 / 4}, & a<0\end{cases}
$$

we get

$$
x_{1}^{\prime}=y_{1}, \quad y_{1}^{\prime}= \pm x_{1}+b y_{1}+y_{1}^{3},
$$

where $b=b / B$ and the prime denotes derivative in $s$. Let $\tau$ be the automorphism defined by (2.5) and $g$ be a Darboux polynomial with cofactor $K=a_{00}+a_{10} x_{1}+a_{01} y_{1}+a_{20} x_{1}^{2}+a_{11} x_{1} y_{1}+a_{02} y_{1}^{2}$ with $a_{i j} \in \mathbb{C}$ for $i, j \in\{0,1,2\}$. In view of Proposition 2.1 we have that $f=g \cdot \tau(g)$ is a Darboux polynomial invariant by $\tau$ and with cofactor $K=2 a_{00}+2 a_{20} x_{1}^{2}+2 a_{11} x_{1} y_{1}+2 a_{02} y_{1}^{2}$. Note that $f\left(x_{1}, y_{1}\right)=f\left(-x_{1},-y_{1}\right)$.

To prove Proposition 3.1 we state and prove the following auxiliary lemma.

Lemma 3.1. System (3.1) has no Darboux polynomials.

Proof of Lemma 9. First, we introduce the weight-change of variables of the form

$$
x_{1}=\alpha^{-3} X, \quad y_{1}=\alpha^{-1} Y, \quad s=\alpha^{2} r .
$$

This type of weight-change of variables to find invariant Darboux polynomial were first introduced in [11]. In this form, system (3.1) becomes

$$
X^{\prime}=\alpha^{4} Y, \quad Y^{\prime}= \pm X+b \alpha^{2} Y+Y^{3},
$$

where now the prime denotes derivative in the new time $r$. Now we set

$$
F(X, Y)=\alpha^{\ell} f\left(\alpha^{-3} X, \alpha^{-1} Y\right)
$$

and

$$
K=2 a_{00}+2 a_{20} \alpha^{-4} X^{2}+2 a_{11} \alpha^{-3} X Y+2 a_{02} \alpha^{-2} Y^{2}
$$

where $\ell$ is the highest weight degree in the weight homogeneous components of $f$ in $X, Y$ with weight $(3,1)$. 
We note that $F=0$ is an invariant algebraic curve of system (3.2) with cofactor $K$. Indeed

$$
\begin{aligned}
\frac{d F}{d r} & =\alpha^{\ell+2} \frac{d f}{d s}=\alpha^{\ell+2}\left(2 a_{00}+2 a_{20} \alpha^{-4} X^{2}+2 a_{11} \alpha^{-3} X Y+2 a_{02} \alpha^{-2} Y^{2}\right) f \\
& =\left(2 a_{02} Y^{2}+2 a_{11} \alpha^{-1} X Y+2 a_{20} \alpha^{-2} X^{2}+2 \alpha^{2} a_{00}\right) F=K F .
\end{aligned}
$$

If $F=\sum_{i=0}^{n} F_{i}$ where $F_{i}$ is a weight homogeneous polynomial in $X, Y$ with weight degree $\ell-i$ for $i=0, \ldots, n$ and $\ell \geq n$, we get

$$
f=\left.F\right|_{\alpha=1} .
$$

From the definition of invariant algebraic curve we have

$$
\begin{aligned}
& \alpha^{4} Y \sum_{i=0}^{n} \alpha^{i} \frac{\partial F_{i}}{\partial X}+\left( \pm X+\alpha^{2} b Y+Y^{3}\right) \sum_{i=0}^{n} \alpha^{i} \frac{\partial F_{i}}{\partial Y} \\
& =\left(2 a_{02} Y^{2}+2 a_{11} \alpha^{-1} X Y+2 a_{20} \alpha^{-2} X^{2}+2 \alpha^{2} a_{00}\right) \sum_{i=0}^{n} \alpha^{i} F_{i} .
\end{aligned}
$$

Equating the terms with $\alpha^{-2}$ in (3.3) we get $a_{20}=0$ and the terms with $\alpha^{-1}$ we get $a_{11}=0$. Moreover, equating the terms with $\alpha^{0}$ in (3.3) we obtain

$$
\left( \pm X+Y^{3}\right) \frac{\partial F_{0}}{\partial Y}=2 a_{02} Y^{2} F_{0}
$$

Since $F_{0} \neq 0$ (otherwise $f$ would be constant) we must have

$$
F_{0}=\beta_{0} X^{n-l}\left( \pm X+Y^{3}\right)^{l}, \quad a_{02}=\frac{3 l}{2},
$$

where $\beta_{0} \neq 0$ (otherwise $f$ would be constant which is not possible). Note that $\ell=3 n$.

Equating now the terms in (3.3) with $\alpha^{1}$ we obtain

$$
\left( \pm X+Y^{3}\right) \frac{\partial F_{1}}{\partial Y}=3 l Y^{2} F_{1} .
$$

Solving it, we get

$$
F_{1}=\tilde{F}_{1}(X)\left( \pm X+Y^{3}\right)^{l}
$$

where $\tilde{F}_{1}$ is a function in $X$. Since $F_{1}$ must be a polynomial of degree $\ell-1=3 n-1$ and $X$ has weight-degree 3 , we must have $\tilde{F}_{1}=0$ and so $F_{1}=0$.

Equating now the terms in (3.3) with $\alpha^{2}$ we get

$$
\left( \pm X+Y^{3}\right) \frac{\partial F_{2}}{\partial Y}+b Y \frac{\partial F_{0}}{\partial Y}=3 l Y^{2} F_{2}+2 a_{00} F_{0} .
$$

Introducing $F_{0}$ we conclude that

$$
\begin{aligned}
F_{2}= & \tilde{F}_{2}(X)\left( \pm X+Y^{3}\right)^{l}+\beta_{0} X^{n-l}\left( \pm X+Y^{3}\right)^{l}\left(\frac{b l}{ \pm X+Y^{3}}\right. \\
& -\frac{\left(2 a_{00}-b l\right)}{\sqrt{3} X^{2 / 3}} \arctan \left(\frac{X^{1 / 3} \mp 2 Y}{\sqrt{3} X^{1 / 3}}\right) \\
& \left.+\frac{2 a_{00}-b l}{3 X^{2 / 3}} \log \left(\frac{\left(X^{1 / 3} \pm Y\right)^{2}}{X^{2 / 3} \mp X^{1 / 3} Y+Y^{2}}\right)\right) .
\end{aligned}
$$


Since $F_{2}$ must be a polynomial with degree $\ell-2=3 n-2$ and $X$ has weight-degree 3 , we must have $\tilde{F}_{2}=0$ and $a_{00}=b l / 2$ (because $\beta_{0} \neq 0$ ). Hence,

$$
f_{n-2}=b \beta_{0} X^{n-l} y\left( \pm X+Y^{3}\right)^{l-1} .
$$

Computing the terms in (3.3) with $\alpha^{3}$ we get

$$
\left( \pm X+Y^{3}\right) \frac{\partial F_{3}}{\partial Y}=3 l Y^{2} F_{3} .
$$

Solving this linear differential equation using that $F_{3}$ has degree $\ell-3=3 n-3$ we get

$$
F_{3}=\beta_{3} X^{n-l-1}\left( \pm X+Y^{3}\right)^{l} .
$$

Computing the terms in (3.3) with $\alpha^{4}$ we obtain

$$
\left( \pm X+Y^{3}\right) \frac{\partial F_{4}}{\partial Y}+b Y \frac{\partial F_{2}}{\partial Y}+Y \frac{\partial F_{0}}{\partial X}=3 l Y^{2} F_{4}+\frac{b l}{2} F_{2} .
$$

Using $F_{2}$ and $F_{0}$ and solving this linear differential equation we obtain

$$
\begin{aligned}
F_{4}= & \tilde{F}_{4}(X)\left( \pm X+Y^{3}\right)^{l} \\
& +\frac{\beta_{0}}{18} X^{n-l}\left( \pm X+Y^{3}\right)^{l}\left(\frac{54 b^{2}(-1+l) l X Y^{2}}{\left( \pm X+Y^{3}\right)^{2}}-\frac{6 l\left(1+6 b^{2} l\right) Y^{2}}{ \pm X+Y^{3}}\right. \\
& +\frac{2 \sqrt{3}\left(6 b^{2} l^{2}+3 n-l\left(2+3 b X^{2 / 3}\right)\right)}{X^{1 / 3}} \arctan \left(\frac{X^{1 / 3} \mp 2 Y}{\sqrt{3} X^{1 / 3}}\right) \\
& \left.+\frac{6 b^{2} l^{2}+3 n-l\left(2+3 b X^{2 / 3}\right)}{X^{1 / 3}} \log \left(\frac{\left(X^{1 / 3} \pm y\right)^{2}}{X^{2 / 3} \mp X^{1 / 3} Y+Y^{2}}\right)\right) .
\end{aligned}
$$

Since $F_{4}$ must be a polynomial of degree $\ell-4=3 n-4$ we must have $\tilde{F}_{4}=0$ and $6 b^{2} l^{2}+3 n-$ $l\left(2+3 b X^{2 / 3}\right)=0$. Since $b \neq 0$ we get $l=n=0$ which is not possible. Hence, there are no Darboux polynomials invariant by $\tau$ and consequently there are no Darboux polynomials.

Now we continue with the proof of Proposition 3.1. Since by Lemma 3.1 system (1.1) with $c=0$ and $a \neq 0$ has no Darboux polynomials, the unique exponential factor of that system may have is of the form $E=\exp (g)$ where $g \in \mathbb{C}[x, y]$ and with cofactor $L \in \mathbb{C}[x, y]$ of the form $L=$ $b_{00}+b_{10} x+b_{01} y+b_{20} x^{2}+b_{11} x y+b_{02} y^{2}$ with $b_{i j} \in \mathbb{C}$ for $i, j \in\{0,1,2\}$. Hence, in view of (2.3) we have

$$
y \frac{\partial g}{\partial x} E+\left(a x+b y+d y^{3}\right) \frac{\partial g}{\partial y} E=L E
$$

and after simplifying by $E=\exp (g)$, we conclude that the polynomial $g$ must satisfy

$$
y \frac{\partial g}{\partial x}+\left(a x+b y+d y^{3}\right) \frac{\partial g}{\partial y}=L .
$$

The divergence of system (1.1) with $c=0$ and $a \neq 0$ is $b+3 d y^{2}$ and in view of Theorem 2.1 we must have $L=b+3 d y^{2}$. So, $g$ must satisfy

$$
y \frac{\partial g}{\partial x}+\left(a x+b y+d y^{3}\right) \frac{\partial g}{\partial y}=L=b+3 d y^{2} .
$$


Evaluating it on $x=y=0$ we get $b=0$. Now we expand $g$ as a polynomial in its homogeneous parts as

$$
g(x, y)=\sum_{j=0}^{n} g_{j}(x, y)
$$

where $g_{j}$ are homogeneous polynomials in $(x, y), n \geq 1$ and $g_{n} \neq 0$. The homogeneous part $g_{n}$ for $n \geq 3$ satisfies

$$
d y^{3} \frac{\partial g_{n}}{\partial y}=0 \text { and so } g_{n}=B_{n} x^{n},
$$

where $B_{n} \in \mathbb{C}$. The homogeneous part $g_{n-1}$ must satisfy

$$
d y^{3} \frac{\partial g_{n-1}}{\partial y}=0 \quad \text { and so } \quad g_{n-1}=B_{n-1} x^{n-1},
$$

where $B_{n-1} \in \mathbb{C}$. Furthermore, the homogeneous part $g_{n-2}$ must satisfy

$$
d y^{3} \frac{\partial g_{n-2}}{\partial y}=B_{n} n x^{n-1} y
$$

and so

$$
g_{n-2}=B_{n-2} x^{n-2}-\frac{B_{n} n}{d y} x^{n-1},
$$

which yields $B_{n}=0$. Hence $g_{n}=0$. This means that $g \in \mathbb{C}[x, y]$ has degree at most two. We write it as

$$
g(x, y)=\lambda_{00}+\lambda_{10} x+\lambda_{01} y+\lambda_{20} x^{2}+\lambda_{11} x y+\lambda_{02} y^{2},
$$

where $\lambda_{i j} \in \mathbb{C}$ for $i, j \in\{0,1,2\}$. Solving (3.4) with such $g$ we get a contradiction. So, in view of Theorems 2.1 and 2.2 it is not Liouvillian integrable. This completes the proof of Proposition 3.1.

\section{Proof of Theorem $1.1(\mathrm{c})$}

First note that if $d c \neq 0$ by the change of coordinates and reparametrization of time of the form

$$
x \rightarrow \frac{1}{C^{1 / 6} d^{1 / 3}} X, \quad y \rightarrow \frac{C^{1 / 6}}{d^{2 / 3}} Y, \quad \tau \rightarrow \frac{C^{2 / 6}}{d^{1 / 3}} t, \quad C=\left\{\begin{array}{ll}
c, & c>0, \\
-c, & c<0
\end{array},\right.
$$

we can write system (1.1) as

$$
\dot{X}=Y, \quad \dot{Y}=\tilde{a} X+\tilde{b} Y+\delta X^{3}+Y^{3}, \quad \delta \in\{-1 ; 1\},
$$

where $\tilde{a}=a d^{2 / 3} / c^{2 / 3}$ and $\tilde{b}=b d^{1 / 3} / c^{1 / 3}$. For convenience in the notation of the paper we will rename system (4.1) as

$$
\dot{x}=y, \quad \dot{y}=a x+b y+\delta x^{3}+y^{3}, \quad \delta \in\{-1 ; 1\} .
$$

The proof of statement (c) of Theorem 1.1 will be a consequence of different results that we will state and prove below. 
Lemma 4.1. The unique irreducible Darboux polynomials of system (4.2) of degrees one or two are:

(i) $f(x, y)=\delta x+y$ with cofactor $K(x, y)=b_{0}+x^{2}-\delta x y+y^{2}$ if $a=\delta b_{0}$ and $b=b_{0}-\delta$ with $b_{0} \in \mathbb{R}$;

(ii) $f(x, y)=1+\delta x^{2}+x y$ with cofactor $K(x, y)=x^{2}-\delta x y+y^{2}$ if $a=1$ and $b=-3 \delta$;

(iii) $f(x, y)=-\delta x^{2}+x y-\delta y^{2}$ with cofactor $K(x, y)=\delta-x^{2}+\delta x y+2 y^{2}$ if $a=-1$ and $b=\delta$.

Proof of Lemma 4.1. It follows by direct computations.

Proposition 4.1. The unique irreducible Darboux polynomials of system (4.2) with degree $n \geq 1$ are the ones given in the statement of Lemma 4.1.

Proof. Let $\tau$ be the automorphism defined by (2.5) and $g$ be a Darboux polynomial with cofactor $K=a_{00}+a_{10} x+a_{01} y+a_{20} x^{2}+a_{11} x y+a_{02} y^{2}$ with $a_{i j} \in \mathbb{C}$ for $i, j \in\{0,1,2\}$. In view of Proposition 2.1 we have that $f=g \cdot \tau(g)$ is a Darboux polynomial invariant by $\tau$ with cofactor $K=2 a_{00}+2 a_{20} x^{2}+2 a_{11} x y+2 a_{02} y^{2}$. Note that $f(x, y)=f(-x,-y)$ and so $f$ has no odd degree terms.

First we want to study the Darboux polynomials of system (4.2) invariant by $\tau$ and after that we will obtain all the Darboux polynomials. Note that if for some values of the parameters there are no Darboux polynomials invariant by $\tau$ then by Proposition 2.1 there are also no Darboux polynomials which may not be invariant by $\tau$. Furthermore, if for some values of the parameters there is a unique irreducible Darboux polynomial invariant by $\tau$ then there are no irreducible Darboux polynomials not invariant by $\tau$ since otherwise in view of Proposition 2.1 the Darboux polynomial not invariant by $\tau$ would be a factor of the Darboux polynomial invariant by $\tau$. But this is not possible since the latter is irreducible. We will see below that the unique irreducible Darboux polynomials invariant by $\tau$ are the ones given in the statement of Lemma 4.1.

By the definition of Darboux polynomial invariant by $\tau$ we have that $f$ and $K$ satisfy

$$
y \frac{\partial f}{\partial x}+\left(a x+b y+\delta x^{3}+y^{3}\right) \frac{\partial f}{\partial y}=\left(a_{00}+a_{20} x^{2}+a_{11} x y+a_{02} y^{2}\right) f
$$

First we expand $f$ as a polynomial in the variable $y$ as

$$
f=\sum_{j=0}^{m} f_{j}(x) y^{j}
$$

Computing the terms with $y^{m+2}$ in (4.3) we get

$$
m f_{m}(x)=a_{02} f_{m}(x) \quad \text { that is } \quad f_{m}(x)\left(a_{02}-m\right)=0,
$$

which yields $a_{02}=m$, that is, $a_{02}$ is an integer which is the greatest power of $f$ in the variable $y$. 
Now we expand $f$ as a polynomial in its homogeneous parts as

$$
f(x, y)=\sum_{j=0}^{n} f_{j}(x, y)
$$

where $f_{j}$ are homogeneous polynomials in $(x, y), n \geq 1$ and $f_{n} \neq 0$. It follows from (4.3) that the homogeneous part $f_{n}$ satisfies

$$
\left(\delta x^{3}+y^{3}\right) \frac{\partial f_{n}}{\partial y}=\left(a_{20} x^{2}+a_{11} x y+a_{02} y^{2}\right) f_{n} .
$$

We will work with $\delta=1$. The case $\delta=-1$ can be done in a similar manner.

Solving (4.4) with $\delta=1$ we get

$$
f_{n}=G_{n}(x)(x+y)^{-\left(a_{11}-a_{20}-a_{02}\right) / 3}\left(x^{2}-x y+y^{2}\right)^{-\left(a_{20}-a_{11}-2 a_{02}\right) / 6}\left(\frac{\sqrt{3} x-i(2 y-x)}{\sqrt{3} x+i(2 y-x)}\right)^{-\sqrt{3}\left(a_{20}+a_{11}\right) i / 6},
$$

where $G_{n}$ is any function in the variable $x$. Note that since $f_{n}$ must be a polynomial and $a_{02}$ is an integer, we must have $a_{20}+a_{11}=0$, i.e., $a_{11}=-a_{20}$ but then we get

$$
f_{n}=G_{n}(x)(x+y)^{\left(2 a_{20}+a_{02}\right) / 3}\left(x^{2}-x y+y^{2}\right)^{\left(a_{02}-a_{20}\right) / 3} .
$$

Again, since $f_{n}$ is a homogeneous polynomial of degree $n$ we have $a_{20}=l_{1}-l_{2}$ and $a_{02}=2 l_{2}+l_{1}$ for some $l_{1}, l_{2} \in \mathbb{N}$ (here $\mathbb{N}$ denotes the set of nonnegative integers) and

$$
f_{n}=A_{n} x^{n-l_{1}-2 l_{2}}(x+y)^{l_{1}}\left(x^{2}-x y+y^{2}\right)^{l_{2}},
$$

for some constant $A_{n} \neq 0$. Without loss of generality we can take $A_{n}=1$. Since $f_{n}(x, y)=$ $\tau\left(f_{n}(x, y)\right)=f_{n}(-x,-y)$ we must have $n$ even.

The homogeneous part $f_{n-1}$ must have degree $n-1$ which is odd and so $f_{n-1}=0$ (because $f$ must be invariant by $\tau$, see the observation at the beginning of the proof of Proposition 4.1). The homogeneous part $f_{n-2}$ must have degree $n-2$ and satisfies

$$
\left(x^{3}+y^{3}\right) \frac{\partial f_{n-2}}{\partial y}+h_{n-2}=\left(a_{20} x^{2}+a_{11} x y+a_{02} y^{2}\right) f_{n-2},
$$

where

$$
h_{n-2}=y \frac{\partial f_{n}}{\partial x}-(a x+2 b y) \frac{\partial f_{n}}{\partial y}-a_{00} f_{n}
$$

Solving it we get

$$
\begin{aligned}
f_{n-2}= & (x+y)^{l_{1}-1}\left(x^{2}-x y+y^{2}\right)^{l_{2}-1}\left[G_{n-2}(x)(x+y)\left(x^{2}-x y+y^{2}\right)\right. \\
& -r_{n-2}(x, y)-s_{1} x^{n-l_{1}-2 l_{2}-2}(x+y)\left(x^{2}-x y+y^{2}\right) \arctan \left(\frac{2 y-x}{\sqrt{3} x}\right) \\
& \left.-s_{2} x^{n-l_{1}-2 l_{2}-2}(x+y)\left(x^{2}-x y+y^{2}\right) \log \left(\frac{x+y}{x^{2}-x y+y^{2}}\right)\right],
\end{aligned}
$$


where $G_{n-2}$ is any function in the variable $x, r_{n-2}(x, y)$ is a polynomial in the variables $x, y$ of degree $n-l_{1}-2 l_{2}+1$ and

$$
\begin{aligned}
& s_{1}=3 a_{00}+(1+a-4 b) l_{1}+(5-a-2 b) l_{2}-3 n, \\
& s_{2}=a_{00}+(a-1) l_{1}-(1+a+2 b) l_{2}+n .
\end{aligned}
$$

Since $f_{n-2}$ must be a homogeneous polynomial of degree $n-2$ and $A_{n} \neq 0$ we must have $G_{n-2}(x)=$ $A_{n-2} x^{n-l_{1}-2 l_{2}-2}$ for some constant $A_{n-2}$ and $s_{1}=s_{2}=0$. Instead of solving $s_{1}=s_{2}=0$ we only save the values of $s_{1}, s_{2}$ and we will solve it later and consider the solution

$$
f_{n-2}=-(x+y)^{l_{1}-1}\left(x^{2}-x y+y^{2}\right)^{l_{2}-1}\left(r_{n-2}(x, y)+A_{n-2} x^{n-l_{1}-2 l_{2}-2}(x+y)\left(x^{2}-x y+y^{2}\right)\right) .
$$

The homogeneous part $f_{n-3}$ must have degree $n-3$ which is odd and so $f_{n-3}=0$ (because $f$ must be invariant by $\tau$ and $f_{n-1}=0$ ). The homogeneous part $f_{n-4}$ must have degree $n-4$ and satisfies

$$
\left(x^{3}+y^{3}\right) \frac{\partial f_{n-4}}{\partial y}+h_{n-4}=\left(a_{20} x^{2}+a_{11} x y+a_{02} y^{2}\right) f_{n-4},
$$

where

$$
h_{n-4}=y \frac{\partial f_{n-2}}{\partial x}-(a x+2 b y) \frac{\partial f_{n-2}}{\partial y}-a_{00} f_{n-2} .
$$

Solving it we get

$$
\begin{aligned}
f_{n-4}= & (x+y)^{l_{1}-2}\left(x^{2}-x y+y^{2}\right)^{l_{2}-2}\left[G_{n-4}(x)(x+y)^{2}\left(x^{2}-x y+y^{2}\right)^{2}\right. \\
& -r_{n-4}(x, y)-s_{3} x^{n-l_{1}-2 l_{2}-4}(x+y)^{2}\left(x^{2}-x y+y^{2}\right)^{2} \arctan \left(\frac{2 y-x}{\sqrt{3} x}\right) \\
& \left.-s_{4} x^{n-l_{1}-2 l_{2}-4}(x+y)^{2}\left(x^{2}-x y+y^{2}\right)^{2} \log \left(\frac{x+y}{x^{2}-x y+y^{2}}\right)\right],
\end{aligned}
$$

where $G_{n-4}$ is any function in the variable $x, r_{n-4}(x, y)$ is a polynomial in the variables $x, y$ of degree $n-l_{1}-2 l_{2}+2$ and $s_{3}, s_{4}$ are very big and we do not write them here. Since $f_{n-4}$ must be a homogeneous polynomial of degree $n-4$ we must have $s_{3}=s_{4}=0$ and then we write it as

$$
f_{n-4}=-(x+y)^{l_{1}-2}\left(x^{2}-x y+y^{2}\right)^{l_{2}-2}\left(r_{n-4}(x, y)+A_{n-4} x^{n-l_{1}-2 l_{2}-4}(x+y)^{2}\left(x^{2}-x y+y^{2}\right)^{2}\right),
$$

for some constant $A_{n-4}$. The homogeneous part $f_{n-5}$ must have degree $n-5$ which is odd and so $f_{n-5}=0$ (because $f$ must be invariant by $\tau$ and $f_{n-3}=0$ ). The homogeneous part $f_{n-6}$ must have degree $n-6$ and satisfies

$$
\left(x^{3}+y^{3}\right) \frac{\partial f_{n-6}}{\partial y}+h_{n-6}=\left(a_{20} x^{2}+a_{11} x y+a_{02} y^{2}\right) f_{n-6},
$$

where

$$
h_{n-6}=y \frac{\partial f_{n-4}}{\partial x}-(a x+2 b y) \frac{\partial f_{n-4}}{\partial y}-a_{00} f_{n-4} .
$$


Solving it we get

$$
\begin{aligned}
f_{n-6}= & (x+y)^{l_{1}-3}\left(x^{2}-x y+y^{2}\right)^{l_{2}-3}\left[G_{n-6}(x)(x+y)^{3}\left(x^{2}-x y+y^{2}\right)^{3}\right. \\
& -r_{n-6}(x, y)-s_{5} x^{n-l_{1}-2 l_{2}-6}(x+y)^{3}\left(x^{2}-x y+y^{2}\right)^{3} \arctan \left(\frac{2 y-x}{\sqrt{3} x}\right) \\
& \left.-s_{6} x^{n-l_{1}-2 l_{2}-6}(x+y)^{3}\left(x^{2}-x y+y^{2}\right)^{3} \log \left(\frac{x+y}{x^{2}-x y+y^{2}}\right)\right],
\end{aligned}
$$

where $G_{n-6}$ is any function in the variable $x, r_{n-6}(x, y)$ is a polynomial in the variables $x, y$ of degree $n-l_{1}-2 l_{2}+3$ and $s_{5}, s_{6}$ are very big and we do not write them here. Since $f_{n-6}$ must be a homogeneous polynomial of degree $n-6$ we must have $s_{5}=s_{6}=0$. Now we solve the system

$$
s_{1}=s_{2}=s_{3}=s_{4}=s_{5}=s_{6}=0
$$

with $n \geq 1$ (an integer) and $l_{1}^{2}+l_{2}^{2} \neq 0$ (the case $l_{1}=l_{2}=0$ yields $n=0$ which is not possible) yield the solutions:

(i) $a=-1, b=1, l_{1}=0, n=2 l_{2}$ and $a_{00}=l_{2}$;

(ii) $a=5 / 2, b=-9 / 2, l_{2}=0, n=3 l_{1}$ and $a_{00}=l_{1} / 2$;

(iii) $a=1, b=-3, l_{2}=0, n=2 l_{1}$ and $a_{00}=0$;

(iv) $b=a-1, l_{2}=0, n=l_{1}$ and $a_{00}=a l_{1}$.

Recall that we are only interested in solutions with $a$ and $b$ real. Note that the case in which the cofactor is zero we have, in particular, that $l_{1}=l_{2}=0$ and so it is not possible because this implies $n=0$. Now we study each of the cases (i)-(iv) separately in different lemmas.

Lemma 4.2. The unique irreducible Darboux polynomial satisfying (i) is the one given in Lemma 4.1 (iii).

Proof of Lemma 4.2. The origin is always a singular point of system (4.2) whose Jacobian matrix at that point has eigenvalues $\left(b \pm \sqrt{b^{2}+4 a}\right) / 2$. Under the assumptions $a=-1, b=1$ the origin is a focus and the eigenvalues of the Jacobian matrix at that point are $(1 \pm \sqrt{3} i) / 2$. Note that they are rationally independent. Moreover, the cofactor of the irreducible Darboux polynomial (if it exists) is of the form

$$
K=K(x, y)=l_{2}\left(1-x^{2}+x y+2 y^{2}\right) .
$$

In particular, $K(0,0)=l_{2} \neq 0$ because $n=2 l_{2} \neq 0$. So, by Proposition 2.2 we must have

$$
l_{2} \in\left\{1, \frac{1+\sqrt{3} i}{2}, \frac{1-\sqrt{3} i}{2}\right\} .
$$

Taking into account that $l_{2}$ is an integer we conclude that $l_{2}=1$ and then $n=2$. We thus conclude that the unique Darboux polynomial invariant by $\tau$ satisfying (i) must have degree two and so it must be the one in Lemma 4.1 (iii). By the observation at the beginning of the proof of Proposition 4.1 we conclude that if there are irreducible Darboux polynomials that are not invariant by $\tau$ must be divisors of the Darboux polynomial $-\delta x^{2}+x y-\delta y^{2}$ but this is not possible because they would have degree one and there are none for these values of the parameters. Hence, the only irreducible Darboux polynomial must be invariant by $\tau$ and so is the one given in Lemma 4.1 (iii). 
Lemma 4.3. There are no irreducible Darboux polynomials satisfying (ii).

Proof of Lemma 4.3. The origin is always a singular point of system (4.2) whose Jacobian matrix at that point has eigenvalues $\left(b \pm \sqrt{b^{2}+4 a}\right) / 2$. Under the assumptions $a=5 / 2, b=-9 / 2$ the origin is a saddle and the eigenvalues of the Jacobian matrix at that point are $-5,1 / 2$. Moreover, the cofactor of the irreducible Darboux polynomial (if it exists) is of the form

$$
K=K(x, y)=l_{1}\left(\frac{1}{2}+x^{2}-x y+y^{2}\right) .
$$

In particular, $K(0,0)=l_{1} / 2 \neq 0$ because $n=3 l_{1} \neq 0$. So, by Proposition 2.2 we must have

$$
\frac{l_{1}}{2} \in\left\{\frac{1}{2},-5,-\frac{9}{2}\right\}
$$

Taking into account that $l_{1}$ is a positive integer we conclude that $l_{1}=1$ and then $n=3$. We thus conclude that the unique irreducible Darboux polynomial satisfying (iii) must have degree three. Computing it we see that it does not exist. Hence, there are no Darboux polynomials invariant by $\tau$. By the observation at the beginning of the proof of Proposition 4.1 we conclude that in fact there are no Darboux polynomials in this case.

Lemma 4.4. The unique irreducible Darboux polynomial satisfying (iii) is the one given in Lemma 4.1 (ii).

Proof of Lemma 4.4. We proceed by contradiction. If we denote by $\phi(x, y)$ the irreducible Darboux polynomial of Lemma 4.1 (ii), that is, $\phi(x, y)=1-x^{2}+x y$ and set $\Phi(x, y)=\phi^{l_{1}}$ then $\Phi$ is a Darboux polynomial of system (4.2) with cofactor $K=l_{1}\left(x^{2}-x y+y^{2}\right)$. Moreover $\Phi$ has degree $n=2 l_{1}$ and if we expand $\Phi$ in its homogeneous terms we get

$$
\Phi(x, y)=x^{l_{1}}(x+y)^{l_{1}}+\sum_{j=0}^{2 l_{1}-2} \Phi_{j}(x, y)
$$

where each $\Phi_{j}$ is a homogeneous polynomial of degree $j$ (we recall that $\Phi$ cannot have odd degree terms). Now define $T=\Phi(x, y)-f(x, y)$ where $f$ is the Darboux polynomial satisfying (iii) with degree $2 l_{1}$. Clearly $T$ is a Darboux polynomial of system (4.2) with cofactor $K=l_{1}\left(x^{2}-x y+y^{2}\right)$. If we expand $T$ in its homogeneous terms we get that $T_{2 l_{1}}=0$ and

$$
T=\sum_{j=0}^{2 l_{1}-2} T_{j}(x, y),
$$

where again each $T_{j}$ is a homogeneous polynomial of degree $j$. Using that $T$ is a Darboux polynomial we get that

$$
T_{2 l_{1}-2}=A_{2 l_{1}-2} x^{l_{1}-2}(x+y)^{l_{1}}, \quad A_{2 l_{1}-2} \in \mathbb{C} .
$$

Note that the terms $T_{2 l_{1}-4}$ satisfy

$$
\left(x^{3}+y^{3}\right) \frac{\partial T_{2 l_{1}-4}}{\partial y}+y \frac{\partial T_{2 l_{1}-2}}{\partial x}+x \frac{\partial T_{2 l_{1}-2}}{\partial y}-3 y \frac{\partial T_{2 l_{1}-2}}{\partial y}=l_{1}\left(x^{2}-x y+y^{2}\right) T_{2 l_{1}-4} .
$$


Solving it we get

$$
\begin{aligned}
T_{2 l_{1}-4}= & A_{2 l_{1}-4} x^{2 l_{1}-3}(x+y)^{l_{1}}-A_{2 l_{1}-2} x^{l_{1}-4}(x+y)^{l_{1}-1}\left[l_{1}\right. \\
& \left.+\frac{3}{\sqrt{3}}(x+y) \arctan \left(\frac{2 y-x}{\sqrt{3} x}\right)+(x+y) \log \left(\frac{x^{2}-x y+y^{2}}{(x+y)^{2}}\right)\right] .
\end{aligned}
$$

Since $T_{2 l_{1}-4}$ must be a polynomial we get $A_{2 l_{1}-2}=0$ and so $T_{2 l_{1}-2}=0$. Now proceeding inductively we get $T_{2 l_{1}-2 j}=0$ for $j=0, \ldots, l_{1}$ which yields $T=0$. In short, $f(x, y)=\Phi(x, y)=\phi^{l_{1}}$. Note that $\phi$ is irreducible. Hence, since $f$ is also irreducible we must have $l_{1}=1$ and then $n=2$. We thus conclude that the unique irreducible Darboux polynomial invariant by $\tau$ satisfying (iii) must have degree two and so it must be the one in Lemma 4.1 (i). By the observation at the beginning of the proof of Proposition 4.1 we conclude that the only irreducible Darboux polynomial must be invariant by $\tau$ and so is the one given in Lemma 4.1 (ii).

Lemma 4.5. The unique irreducible Darboux polynomial satisfying (iv) is the one given in Lemma 4.1 (i).

Proof of Lemma 4.5. The proof is similar to the proof of Lemma 4.4. We also proceed by contradiction. Let $\Phi(x, y)=(x+y)^{l_{1}}$. Note that $\Phi$ is a Darboux polynomial of system (4.2) with cofactor $K=l_{1}\left(b_{0}+x^{2}-x y+y^{2}\right)$. Now let $T=\Phi(x, y)-f(x, y)$ where $f$ is the Darboux polynomial satisfying (iv) with degree $l_{1}$. Clearly, $T$ is a Darboux polynomial of system (4.2) with cofactor $K=l_{1}\left(b_{0}+x^{2}-x y+y^{2}\right)$. If we expand $T$ in its homogeneous terms we get $T_{l_{1}}=0$ and

$$
T=\sum_{j=0}^{l_{1}-1} T_{j}(x, y),
$$

where again each $T_{j}$ is a homogeneous polynomial of degree $j$. Using that $T$ is a Darboux polynomial we get

$$
T_{l_{1}-1}=A_{l_{1}-1}(x+y)^{l_{1}-1}, \quad A_{l_{1}-1} \in \mathbb{C} .
$$

Note that the terms $T_{l_{1}-2}$ satisfy

$$
T_{l_{1}-2}=A_{l_{1}-2}(x+y)^{l_{1}-2}, \quad A_{l_{1}-2} \in \mathbb{C} .
$$

Moreover, the terms $T_{l_{1}-3}$ satisfy

$$
\left(x^{3}+y^{3}\right) \frac{\partial T_{l_{1}-3}}{\partial y}+y \frac{\partial T_{l_{1}-3}}{\partial x}+b_{0} x \frac{\partial T_{l_{1}-1}}{\partial y}+\left(b_{0}+1\right) y \frac{\partial T_{l_{1}-1}}{\partial y}-b_{0} l_{1} T_{l_{1}-1}=l_{1}\left(x^{2}+x y+y^{2}\right) T_{l_{1}-3} .
$$

Solving it and using that $T_{l_{1}-3}$ is a polynomial we get $A_{l_{1}-1}=0$ and so $T_{l_{1}-1}=0$. Proceeding inductively we obtain $T=0$ and so $f(x, y)=(x+y)^{l_{1}}$. Since $f$ must be irreducible we get $l_{1}=1$ and $n=1$ so it must be the one of Lemma 4.1 (i).

Proceeding in a similar manner for the case $\delta=-1$ we also obtain that the unique irreducible Darboux polynomials are the ones of degree one and two given in Lemma 4.1.

Proposition 4.2. System (4.2) is not Liouvillian integrable.

Proof. We separate the proof of Proposition 4.2 into different lemmas. 
Lemma 4.6. System (4.2) under the assumptions of Lemma 4.1 (i) is not Liouvillian integrable.

Proof of Lemma 4.6. The unique exponential factor that system (4.2) may have is of the form $E=\exp \left(g /(y+\delta x)^{n}\right)$ where $g \in \mathbb{C}[x, y], n \geq 0$ and $g$ is coprime with $y+\delta x$ whenever $n>0$. Moreover, the cofactor $L \in \mathbb{C}[x, y]$ is of the form $L=b_{00}+b_{10} x+b_{01} y+b_{20} x^{2}+b_{11} x y+b_{02} y^{2}$ where $b_{i j} \in \mathbb{C}$ for $i, j \in\{0,1,2\}$. Hence, in view of(2.3) we have

$$
y \frac{\partial g}{\partial x} E+\left(\delta b_{0} x+\left(b_{0}-\delta\right) y+\delta x^{3}+y^{3}\right) \frac{\partial g}{\partial y} E-n\left(b_{0}+x^{2}-\delta x y+y^{2}\right) g E=L(y+\delta x)^{n} E,
$$

and after simplifying by $E=\exp \left(g /(y+\delta x)^{n}\right)$, we conclude that $g$ must satisfy

$$
y \frac{\partial g}{\partial x}+\left(\delta b_{0} x+\left(b_{0}-\delta\right) y+\delta x^{3}+y^{3}\right) \frac{\partial g}{\partial y}-n\left(b_{0}+x^{2}-\delta x y+y^{2}\right) g=L(y+\delta x)^{n} .
$$

Assume that $n>0$ and so $g$ is coprime with $y+\delta x$. If we denote by $\bar{g}$ the restriction of $g$ to $y=-\delta x$ then $\bar{g} \neq 0$ and satisfies

$$
-\delta x \frac{d \bar{g}}{d x}-n\left(b_{0}+3 x^{2}\right) \bar{g}=0,
$$

because $\delta^{2}=1$. Solving the above linear differential equation we get

$$
\bar{g}(x)=\alpha e^{-3 n x^{2} / 2} x^{-n b_{0} / \delta}, \quad \alpha \in \mathbb{C} .
$$

Since $\bar{g}$ must be a polynomial and $n>0$, we get $\alpha=0$ and so $\bar{g}=0$ which is not possible. So, $n=0$ and $g$ satisfies

$$
y \frac{\partial g}{\partial x}+\left(\delta b_{0} x+\left(b_{0}-\delta\right) y+\delta x^{3}+y^{3}\right) \frac{\partial g}{\partial y}=L .
$$

Note that the divergence of system (4.2) is $b_{0}-\delta+3 y^{2}$ and in view of Theorem 2.1 we must have $L=-b_{0}+\delta-3 y^{2}-\lambda\left(b_{0}+x^{2}-\delta x y+y^{2}\right)$ for some $\lambda \in \mathbb{C}$. Hence, $g$ must satisfy

$$
y \frac{\partial g}{\partial x}+\left(\delta b_{0} x+\left(b_{0}-\delta\right) y+\delta x^{3}+y^{3}\right) \frac{\partial g}{\partial y}=-\left(-b_{0}+\delta-3 y^{2}-\lambda\left(b_{0}+x^{2}-\delta x y+y^{2}\right)\right) .
$$

Evaluating (4.5) on $x=y=0$ we get $\delta=b_{0}(1+\lambda)$. Now we expand $g$ as a polynomial in its homogeneous parts as

$$
g(x, y)=\sum_{j=0}^{m} g_{j}(x, y)
$$

where $g_{j}$ are homogeneous polynomials in $(x, y), m \geq 1$ and $g_{m} \neq 0$. The homogeneous part $g_{m}$ for $m \geq 3$ satisfies

$$
\left(\delta x^{3}+y^{3}\right) \frac{\partial g_{m}}{\partial y}=0 \quad \text { and so } \quad g_{m}=B_{m} x^{m},
$$

where $B_{m} \in \mathbb{C}$. The homogeneous part $g_{m-1}$ must satisfy

$$
\left(\delta x^{3}+y^{3}\right) \frac{\partial g_{m-1}}{\partial y}=0 \quad \text { and so } \quad g_{m-1}=B_{m-1} x^{m-1} .
$$


Furthermore, the homogeneous part $g_{m-2}$ must satisfy

$$
\left(\delta x^{3}+y^{3}\right) \frac{\partial g_{m-2}}{\partial y}=-B_{m} m x^{m-1} y
$$

and so

$$
g_{m-2}=B_{m-2} x^{m-2}-B_{m} \frac{m \delta}{6} x^{m-2}\left[2 \sqrt{3} \arctan \left(\frac{2 y-\delta x}{\sqrt{3} \delta x}\right)+\log \left(\frac{\delta x^{2}-\delta x y+y^{2}}{(x+y)^{2}}\right)\right],
$$

which yields $B_{m}=0$. Hence $g_{m}=0$. This means that $g \in \mathbb{C}[x, y]$ and has degree at most two. We write it as

$$
g(x, y)=\lambda_{00}+\lambda_{10} x+\lambda_{01} y+\lambda_{20} x^{2}+\lambda_{11} x y+\lambda_{02} y^{2},
$$

with $\lambda_{i, j} \in \mathbb{C}$ for $i, j \in\{0,1,2\}$. Solving equation (4.5) with $g$ as above we get a contradiction. So, in view of Theorem 2.1 and 2.2 it is not Liouvillian integrable.

Lemma 4.7. System (4.2) under the assumptions of Lemma 4.1 (ii) is not Liouvillian integrable.

Proof of Lemma 4.7. The unique exponential factor that system (4.2) may have is of the form $E=\exp \left(g /\left(1+\delta x^{2}+x y\right)^{n}\right)$ where $g \in \mathbb{C}[x, y], n \geq 0$ and $g$ is coprime with $1+\delta x^{2}+x y$ whenever $n>0$. Moreover, the cofactor $L \in \mathbb{C}[x, y]$ is of the form $L=b_{00}+b_{10} x+b_{01} y+b_{20} x^{2}+b_{11} x y+b_{02} y^{2}$ where $b_{i j} \in \mathbb{C}$ for $i, j \in\{0,1,2\}$. Hence, in view of (2.3) we have

$$
y \frac{\partial g}{\partial x} E+\left(x-3 \delta y+\delta x^{3}+y^{3}\right) \frac{\partial g}{\partial y} E-n\left(x^{2}-\delta x y+y^{2}\right) g E=L\left(1+\delta x^{2}+x y\right)^{n} E,
$$

and after simplifying by $E=\exp \left(g /\left(1+\delta x^{2}+x y\right)^{n}\right)$, we conclude that $g$ must satisfy

$$
y \frac{\partial g}{\partial x}+\left(x-3 \delta y+\delta x^{3}+y^{3}\right) \frac{\partial g}{\partial y}-n\left(x^{2}-\delta x y+y^{2}\right) g=L\left(1+\delta x^{2}+x y\right)^{n} .
$$

Note that the divergence of system (4.2) is $-3 \delta+3 y^{2}$ and in view of Theorem 2.1 we must have $L=3 \delta-3 y^{2}-\lambda\left(x^{2}-\delta x y+y^{2}\right)$ for some $\lambda \in \mathbb{C}$, and so

$$
\begin{aligned}
& y \frac{\partial g}{\partial x}+\left(x-3 \delta y+\delta x^{3}+y^{3}\right) \frac{\partial g}{\partial y}-n\left(x^{2}-\delta x y+y^{2}\right) g \\
& =\left(3 \delta-3 y^{2} \lambda\left(x^{2}-\delta x y+y^{2}\right)\right)\left(1+\delta x^{2}+x y\right)^{n} .
\end{aligned}
$$

Evaluating (4.6) on $x=y=0$ we get $0=3 \delta$ which is not possible. Therefore, in this case there is no such an exponential factor. This concludes the proof.

Lemma 4.8. System (4.2) under the assumptions of Lemma 4.1 (iii) is not Liouvillian integrable.

Proof of Lemma 4.8. The unique exponential factor that system (4.2) may have is of the form $E=\exp \left(g /\left(-\delta x^{2}+x y-\delta y^{2}\right)^{n}\right)$ where $g \in \mathbb{C}[x, y], n \geq 0$ and $g$ is coprime with $-\delta x^{2}+x y-\delta y^{2}$ whenever $n>0$. Moreover, the cofactor $L \in \mathbb{C}[x, y]$ is of the form $L=b_{00}+b_{10} x+b_{01} y+b_{20} x^{2}+$ 
$b_{11} x y+b_{02} y^{2}$ where $b_{i j} \in \mathbb{C}$ for $i, j \in\{0,1,2\}$. Hence, in view of (2.3) we have

$$
y \frac{\partial g}{\partial x} E+\left(-x+\delta y+\delta x^{3}+y^{3}\right) \frac{\partial g}{\partial y} E-n\left(\delta-x^{2}+\delta x y+2 y^{2}\right) g E=L\left(-\delta x^{2}+x y-\delta y^{2}\right)^{n} E,
$$

and after simplifying by $E=\exp \left(g /\left(-\delta x^{2}+x y-\delta y^{2}\right)^{n}\right)$, we conclude that $g$ must satisfy

$$
y \frac{\partial g}{\partial x}+\left(-x+\delta y+\delta x^{3}+y^{3}\right) \frac{\partial g}{\partial y}-n\left(\delta-x^{2}+\delta x y+y^{2}\right) g=L\left(-\delta x^{2}+x y-\delta y^{2}\right)^{n} .
$$

Note that the divergence of system (4.2) is $\delta+3 y^{2}$ and by Theorem 2.1 we must have $L=-\delta-$ $3 y^{2}-\lambda\left(\delta-x^{2}+\delta x y+y^{2}\right)$ for some $\lambda \in \mathbb{C}$. So, by (4.7) $g$ must satisfy

$$
\begin{aligned}
& y \frac{\partial g}{\partial x}+\left(-x+\delta y+\delta x^{3}+y^{3}\right) \frac{\partial g}{\partial y}-n\left(\delta-x^{2}+\delta x y+y^{2}\right) g \\
& =-\left(\delta+3 y^{2}-\lambda\left(\delta-x^{2}+\delta x y+y^{2}\right)\right)\left(-\delta x^{2}+x y-\delta y^{2}\right)^{n} .
\end{aligned}
$$

Evaluating (4.8) on $y=0$ and $x= \pm \sqrt{\delta}$ we get $0=(-1)^{n+1} \delta^{3 n+1}$ which is not possible. Hence, in this case there is no such an exponential factor. This concludes the proof.

Lemma 4.9. System (4.2) under none of the assumptions of Lemma 4.1 is not Liouvillian integrable.

Proof of Lemma 4.9. Since system (4.2) has no Darboux polynomial, so the unique exponential factor that system (4.2) may have is of the form $E=\exp (g)$ where $g \in \mathbb{C}[x, y]$ and with cofactor $L \in$ $\mathbb{C}[x, y]$ of the form $L=b_{00}+b_{10} x+b_{01} y+b_{20} x^{2}+b_{11} x y+b_{02} y^{2}$ where $b_{i j} \in \mathbb{C}$ for $i, j \in\{0,1,2\}$. Hence, in view of (2.3) we have

$$
y \frac{\partial g}{\partial x} E+\left(a x+b y+\delta x^{3}+y^{3}\right) \frac{\partial g}{\partial y} E=L E
$$

and after simplifying by $E=\exp (g)$, we conclude that $g$ must satisfy

$$
y \frac{\partial g}{\partial x}+\left(a x+b y+\delta x^{3}+y^{3}\right) \frac{\partial g}{\partial y}=L
$$

Note that the divergence of system (4.2) is $b+3 y^{2}$ and in view of Theorem 2.1 we must have $L=-b-3 y^{2}$. So, in view of (4.9), $g$ must satisfy

$$
y \frac{\partial g}{\partial x}+\left(a x+b y+\delta x^{3}+y^{3}\right) \frac{\partial g}{\partial y}=L=-b-3 y^{2} .
$$

Evaluating it on $x=y=0$ we get $b=0$. Now we expand $g$ as a polynomial in its homogeneous parts as $g(x, y)=\sum_{j=0}^{n} g_{j}(x, y)$, where $g_{j}$ are homogeneous polynomials in $(x, y), n \geq 1$ and $g_{n} \neq 0$. 
The homogeneous part $g_{n}$ for $n \geq 3$ satisfies

$$
\left(\delta x^{3}+y^{3}\right) \frac{\partial g_{n}}{\partial y}=0 \quad \text { and so } \quad g_{n}=B_{n} x^{n}
$$

where $B_{n} \in \mathbb{C}$. The homogeneous part $g_{n-1}$ must satisfy

$$
\left(\delta x^{3}+y^{3}\right) \frac{\partial g_{n-1}}{\partial y}=0 \quad \text { and so } \quad g_{n-1}=B_{n-1} x^{n-1},
$$

where $B_{n-1} \in \mathbb{C}$. Furthermore, the homogeneous part $g_{n-2}$ must satisfy

$$
\left(\delta x^{3}+y^{3}\right) \frac{\partial g_{n-2}}{\partial y}=-B_{n} n x^{n-1} y
$$

and so

$$
g_{n-2}=B_{n-2} x^{n-2}-B_{n} \frac{n \delta}{6} x^{n-2}\left[2 \sqrt{3} \arctan \left(\frac{2 y-\delta x}{\sqrt{3} \delta x}\right)+\log \left(\frac{\delta x^{2}-\delta x y+y^{2}}{(x+y)^{2}}\right)\right],
$$

which yields $B_{n}=0$. Hence $g_{n}=0$. This means that $g \in \mathbb{C}[x, y]$ has degree at most two. We write it as

$$
g(x, y)=\lambda_{00}+\lambda_{10} x+\lambda_{01} y+\lambda_{20} x^{2}+\lambda_{11} x y+\lambda_{02} y^{2},
$$

with $\lambda_{i, j} \in \mathbb{C}$ for $i, j \in\{0,1,2\}$. Solving equation (4.10) with $g$ as above we get a contradiction. So, in view of Theorem 2.1 and 2.2 it is not Liouvillian integrable.

Now, the proof of Proposition 4.2 follows directly from Lemmas 4.6-4.9.

\section{Acknowledgements}

The authors are grateful to the referees for their valuable comments and suggestions to improve this paper. The first author is partially supported by a MINECO/ FEDER grant number MTM201784383-P and an AGAUR (Generalitat de Catalunya) grant number 2017SGR-1276. The second author is supported by FCT/Portugal through UID/MAT/04459/2013.

\section{References}

[1] J. Chavarriga, H. Giacomini, J. Giné, J. Llibre, On the integrability of two-dimensional flows, J. Differential Equations 157 (1999), no. 1, 163-182.

[2] J. Chavarriga, H. Giacomini, M. Grau, Necessary conditions for the existence of invariant algebraic curves for planar polynomial systems, Bull. Sci. Math. 129, (2005), 99-126.

[3] H. Chen, L. Zou, Global study of Rayleigh-Duffing oscillators, J. Phys. A 49 (2016), no. 16, 165202.

[4] M.V. Demina, Novel algebraic aspects of Liouvillian integrability for two-dimensional dynamical systems, Phys. Lett. A 382 (2018), no. 20, 1353-1360.

[5] F. Dumortier, J. Llibre, J.C. Artés, Qualitative Theory of Planar Differential Systems, Springer Verlag, New York, 2006.

[6] J. Giné, C. Valls, On the dynamics of the Rayleigh-Duffing oscillator, Nonlinear Anal. Real World Appl. 45 (2019), 309-319.

[7] Y. Kanai, H. Yabuno, Creation-annihilation process of limit cycles in the Rayleigh-Duffing oscillator, Nonlinear Dynam. 70 (2012), no. 2, 1007-1016. 
[8] D.W. Jordan, P. Smith, Nonlinear ordinary differential equations - An introduction for scientists and engineers (4th ed.), Oxford University Press, 2007.

[9] W. Li, J. Llibre, X. Zhang, On the differentiability of first integrals of two dimensional flows, Proc. Amer. Math. Soc. 130 (2002), 2079-2088.

[10] W. Li, J. Llibre, X. Zhang, Local first integrals of differential systems and diffeomorphisms, Z. Angew. Math. Phys. 54 (2003), 235-255.

[11] J. Llibre, X. Zhang, Invariant algebraic surfaces of the Lorenz system, J. Math. Phys. 43 (2002), no. 3, $1622-1645$.

[12] H. Poincaré, Mémoire sur les courbes définies par les équations différentielles, Euvres de Henri Poincaré, vol. I Gauthier-Villars, Paris (1951), 95-114.

[13] H. Poincaré, Sur l'intégration algébrique des équations différentielles du premier ordre et du premier degrée, Rend. Circ. Mat. Palermo 5 (1891), 161-191; 11 (1897), 193-239.

[14] M.F. Singer, Liouvillian first integrals of differential equations, Trans. Am. Math. Soc. 333 (1992), 673-688.

[15] J.M. Strutt, Scientific Papers, Macmillan and Co., London, 1943. 